\title{
Lower bounds of end effects for a non homogeneous isotropic linear elastic solid in anti-plane shear
}

\author{
M.C. Leseduarte and R. Quintanilla \\ Matemàtica Aplicada 2 Universitat Politècnica de Catalunya \\ Colom, 11. Terrassa. Barcelona. Spain \\ E-mail: mari.carme.leseduarte@upc.edu,ramon.quintanilla@upc.edu
}

Dedicated to K.R. Rajagopal with great esteem

\begin{abstract}
In this paper we give lower bounds for the spatial decay of the solutions for anti-plane shear deformations in the case of isotropic inhomogeneous elastic materials. We first consider the case when the shear modulus only depends on the lateral direction. By means of the logarithmic convexity arguments we obtain the required estimates. Some pictures illustrate our results. Last section studies the general inhomogeneity. We give some lower bounds whenever shear modulus satisfies several requirements.
\end{abstract}

keywords: Spatial decay rates, inhomogeneous boundary problems, lower bounds, anti-plane shear deformations, logarithmic convexity argument

\section{Introduction}

The study of spatial decay estimates for partial differential equations has received much attention in recent years. This is due among other things to its relationship with the so-called Saint Venant's principle. For these studies it is usual to consider a semi-infinite cylinder or strip and applying a small perturbation in the finite end of the cylinder (or strip). It is customary to study the damping of displacement when we move far away from the border where the disturbance is applied. From a mathematical point of view, we consider a functional of the solution and we see how it decays when the spatial variable grows to infinity. More often the functional is superiorly bounded by a negative exponential decay function or another kind of decaying function. This gives us a greater extent to decay, but there are no contributions in the literature which give us lower bounds on the decay of solutions. This alternative work is also relevant because it gives us a spatial decay information. Upper bounds control how great may be the decay and by using lower bounds we get a measure of what we cannot dismiss of the solution. In this manuscript we intend to make a first contribution in this line and get lower bounds for the decay of the solutions. We develop this task for the case of anti-plane shear deformations for functionally graduated linear elastic materials.

We want to obtain estimates for the lower bound of the decay of solutions and, in particular, we want to emphasize how the material inhomogeneity affects to the lower bound of the decay of solutions. The effects of the material inhomogeneity on the decay of solutions for boundary value problems on a semi-infinite strip has been considered in recents years. Scalpato and Horgan [12], Chan and Horgan [4], Horgan and Payne [6] Horgan and Quintanilla [7, 8] and Borrelli et al. [3] obtained upper bounds for the decay of solutions and Leseduarte and Quintanilla $[9,10]$ extended some of these arguments to the case of mixtures. In these contributions, the authors showed how the material inhomogeneity can have a significant influence on the decay of end effects. 
The motivation for these studies has been provided by the research activity on functionally graded materials (FGMs), that is, materials with continuously varying properties tailored to satisfy specific engineering applications (see, e.g. the papers by Erdogan [5], Pindera et al. [11] and Aboudi et al. [1] and the references cited therein). We believe that obtaining lower bounds of the solutions for functionally graded materials is a task which deserves to be analyzed.

In this paper we study lower bounds for the decay of the solutions of the problem determined by the equation

$$
\left[\mu\left(x_{1}, x_{2}\right) u_{, i}\right]_{, i}=0,
$$

defined on the semi-infinite strip $(0, \infty) \times(0, L)$ when we impose the boundary conditions

$$
u\left(x_{1}, 0\right)=u\left(x_{1}, L\right)=0, x_{1} \geq 0 \text { and } u\left(0, x_{2}\right)=f\left(x_{2}\right), x_{2} \in[0, L],
$$

where $f\left(x_{2}\right)$ is sufficiently smooth and satisfies $f(0)=f(L)=0$. The shear modulus is $\mu\left(x_{1}, x_{2}\right)$. We will suppose $\mu\left(x_{1}, x_{2}\right) \geq 0$. Finally, we assume the asymptotic conditions

$$
u \rightarrow 0 \text { and } u_{, i} \rightarrow 0 \text { as } x_{1} \rightarrow \infty \text { (uniformly on } x_{2} \text { ). }
$$

It is worth noting that in the case of homogeneous materials, the equation (1.1) becomes the Laplace equation. The problem determined with prescribed displacement at the finite edge is well known. The solutions can be obtained by means of the separation of variables method.

The plan of this paper is: in Section 2 we consider the case when the homogeneity only depends on the lateral direction. We obtain a lower estimate for the decay. This decay depends strongly on the boundary condition $f\left(x_{2}\right)$. Thus, to obtain the lower bounds we need to consider the particular boundary condition. We illustrate it by examples in Section 3. Section 4 is devoted to the case of a general inhomogeneity. We also give lower estimates for the decay whenever the shear modulus satisfies several requirements.

\section{Case $\mu=\mu\left(x_{2}\right)$}

In this section we study lower decay rates for solutions of the problem (1.1)-(1.3) when the function $\mu$ does not depend on the variable $x_{1}$. That is, $\mu=\mu\left(x_{2}\right)$ on $[0, L]$. Moreover, we assume $\mu\left(x_{2}\right) \geq 0$. In this case, the material inhomogeneity varies in the lateral direction only. The equation (1.1) becomes

$$
\left[\mu\left(x_{2}\right) u_{, i}\right]_{, i}=0 .
$$

In this section we obtain several lower bounds for the solutions in the case where $\mu\left(x_{2}\right) \geq 0$ and $f\left(x_{2}\right) \in$ $H^{1,2}[0, L]$ As a consequence, the impossibility of localization of solutions will be also proved.

We define the following measure on the solutions

$$
F\left(x_{1}\right)=\frac{1}{2} \int_{0}^{L} \mu\left(x_{2}\right) u^{2} d x_{2} .
$$

Then, we have that

$$
F^{\prime}\left(x_{1}\right)=\int_{0}^{L} \mu\left(x_{2}\right) u u_{, 1} d x_{2} \text { and } F^{\prime \prime}\left(x_{1}\right)=\int_{0}^{L} \mu\left(x_{2}\right)\left(u_{, 1}^{2}+u u_{, 11}\right) d x_{2} .
$$

If we multiply the equation (2.1) by $u$ and we integrate over $[0, L]$, we obtain

$$
F^{\prime \prime}\left(x_{1}\right)=\int_{0}^{L} \mu\left(x_{2}\right)\left(u_{, 1}^{2}+u_{, 2}^{2}\right) d x_{2}
$$


We need an equality which relates the integrals of the partial derivatives of $u$. Now, we multiply the equation (2.1) by $u_{, 1}$. After integration over $\left[0, x_{1}\right] \times[0, L]$ we obtain the "energy equation"

$$
E\left(x_{1}\right)=\frac{1}{2} \int_{0}^{L} \mu\left(x_{2}\right)\left(u_{, 1}^{2}-u_{, 2}^{2}\right) d x_{2}=E(0) \quad(=0) .
$$

We note that $E\left(x_{1}\right)$ vanishes because the asymptotic conditions (1.3). From (2.4) and (2.5) we obtain that

$$
F^{\prime \prime}\left(x_{1}\right)=2 \int_{0}^{L} \mu\left(x_{2}\right) u_{, 1}^{2} d x_{2}
$$

As

$$
F F^{\prime \prime}-\left(F^{\prime}\right)^{2}=\left(\int_{0}^{L} \mu\left(x_{2}\right) u^{2} d x_{2}\right)\left(\int_{0}^{L} \mu\left(x_{2}\right) u_{, 1}^{2} d x_{2}\right)-\left(\int_{0}^{L} \mu\left(x_{2}\right) u u_{1,1} d x_{2}\right)^{2},
$$

we can use the Hölder inequality to see that $F F^{\prime \prime}-\left(F^{\prime}\right)^{2} \geq 0$. So, $\ln F\left(x_{1}\right)$ is a convex function. We get that (see [2], p.19)

$$
F\left(x_{1}\right) \geq F(0) \exp \left[\left(F^{\prime}(0) / F(0)\right) x_{1}\right] .
$$

From the estimate (2.8) we see that the decay is lower controlled by $\exp \left[\left(F^{\prime}(0) / F(0)\right) x_{1}\right]$. Thus, to have a good description of the decay, we need to have a lower bound for the quotient $F^{\prime}(0) / F(0)$ in terms of the data of the problem. We have

$$
\begin{aligned}
-F^{\prime}(0) & \leq\left(\int_{0}^{L} \mu\left(x_{2}\right) u^{2}\left(0, x_{2}\right) d x_{2}\right)^{1 / 2}\left(\int_{0}^{L} \mu\left(x_{2}\right) u_{, 1}^{2}\left(0, x_{2}\right) d x_{2}\right)^{1 / 2} \\
& =\left(\int_{0}^{L} \mu\left(x_{2}\right) u^{2}\left(0, x_{2}\right) d x_{2}\right)^{1 / 2}\left(\int_{0}^{L} \mu\left(x_{2}\right) u_{, 2}^{2}\left(0, x_{2}\right) d x_{2}\right)^{1 / 2}
\end{aligned}
$$

where the equality follows from the energy equation. Thus, we see that

$$
-\frac{F^{\prime}(0)}{F(0)} \leq 2\left(\int_{0}^{L} \mu\left(x_{2}\right) u_{, 2}^{2}\left(0, x_{2}\right) d x_{2} / \int_{0}^{L} \mu\left(x_{2}\right) u^{2}\left(0, x_{2}\right) d x_{2}\right)^{1 / 2} .
$$

Therefore,

$$
F\left(x_{1}\right) \geq F(0) \exp \left[-2\left(\int_{0}^{L} \mu\left(x_{2}\right)\left(f^{\prime}\left(x_{2}\right)\right)^{2} d x_{2} / \int_{0}^{L} \mu\left(x_{2}\right) f^{2}\left(x_{2}\right) d x_{2}\right)^{1 / 2} x_{1}\right] .
$$

We have proved:

Theorem 2.1 Let $u\left(x_{1}, x_{2}\right)$ be a solution of the problem (1.1)-(1.3). Assume that $\mu=\mu\left(x_{2}\right) \geq 0$. Then, estimate (2.11) is satisfied, where $F\left(x_{1}\right)$ is defined by (2.2).

If we assume that $\mu\left(x_{2}\right)$ can be zero ${ }^{1}$ at most in a subset of measure zero, then the only possibility to have

$$
\int_{0}^{L} \mu\left(x_{2}\right) f^{2}\left(x_{2}\right) d x_{2}=0
$$

is that $f\left(x_{2}\right) \equiv 0$. If we also assume that $\int_{0}^{L} \mu\left(x_{2}\right)\left(f^{\prime}\left(x_{2}\right)\right)^{2} d x_{2}<+\infty$, we see that right hand side of (2.11) cannot vanish. Thus, we have proved:

\footnotetext{
${ }^{1}$ When the function $\mu\left(x_{2}\right)$ vanishes at $x_{2}=0$, we relax the boundary condition to assume that $f(0)$ is bounded.
} 
Corollary 2.1 Let us to assume that $\mu\left(x_{2}\right)$ can only be zero in a subset of measure zero and $f\left(x_{2}\right) \in H_{\mu}^{1,2}[0, L]$. The only solution $u\left(x_{1}, x_{2}\right)$ for the problem (1.1)-(1.3) which can be identically zero after a finite value $x_{1} \geq$ $x_{1}^{0} \geq 0$ is the null solution.

Let us consider the Sturm-Liouville problem

$$
\left(\mu\left(x_{2}\right) y^{\prime}\right)^{\prime}+\lambda \mu\left(x_{2}\right) y=0, \quad y(0)=y(L)=0
$$

and let us to assume that it admits a sequence of normalized eigenfunctions $y_{1}\left(x_{2}\right), y_{2}\left(x_{2}\right), \ldots, y_{n}\left(x_{2}\right) \ldots$ with eigenvalues $\lambda_{1}<\lambda_{2}<\ldots<\lambda_{n}<\ldots$ and such that the function $f\left(x_{2}\right)$ can be expressed as

$$
f\left(x_{2}\right)=\sum_{n=1}^{\infty} c_{n} y_{n}\left(x_{2}\right) \text {. }
$$

Then, the following estimate

$$
F\left(x_{1}\right) \geq F(0) \exp \left[-2\left(\sum_{n=1}^{\infty} \lambda_{n} c_{n}^{2} / \sum_{n=1}^{\infty} c_{n}^{2}\right)^{1 / 2} x_{1}\right]
$$

is satisfied. The convergence of the series appearing here is guaranteed by the fact that $f\left(x_{2}\right) \in H_{\mu}^{1,2}[0, L]$. We have obtained:

Corollary 2.2 Let us to assume that $f\left(x_{2}\right)$ can be written in the form (2.14), where $y_{n}\left(x_{2}\right)$ is the sequence of the eigenfunctions of the problem (2.13). Then, the estimate (2.15) is satisfied.

It is not easy to have a good knowledge of the eigenvalues and eigenfunctions of the problem (2.13). However, there is a family of functions $\mu\left(x_{2}\right)$ such that it is possible to find the sequence of eigenvalues and eigenfunctions by means of a change of variables. Let us assume that

$$
\left(\mu^{1 / 2}\right)^{\prime \prime}=c \mu^{1 / 2}
$$

where $c$ is a constant. If we consider the function

$$
w\left(x_{2}\right)=\mu^{1 / 2}\left(x_{2}\right) y\left(x_{2}\right),
$$

we see that the function $w$ satisfies the problem

$$
w^{\prime \prime}\left(x_{2}\right)+(\lambda-c) w\left(x_{2}\right)=0, \quad w(0)=w(L)=0 .
$$

Thus, $\lambda_{1}<\lambda_{2}<\ldots<\lambda_{n}<\ldots$ is the sequence of eigenvalues of the problem (2.13) if and only if $\lambda_{1}-c<$ $\lambda_{2}-c<\ldots<\lambda_{n}-c<\ldots$ is the sequence of eigenvalues of the problem

$$
w^{\prime \prime}\left(x_{2}\right)+\lambda w\left(x_{2}\right)=0, \quad w(0)=w(L)=0 .
$$

In fact, the eigenfunctions are

$$
y\left(x_{2}\right)=\mu^{-1 / 2}\left(x_{2}\right) \sin \left(n \pi x_{2} / L\right)
$$

and $\lambda_{n}=(n \pi / L)^{2}+c$. We can separate three different cases. In all of them we assume that $L=\pi$.

(a) Case $c>0$. We have $\mu^{1 / 2}\left(x_{2}\right)=\mu_{0}^{1 / 2} \exp \left( \pm \alpha x_{2}\right)$, with $\alpha=c^{1 / 2}$. Thus, $\mu\left(x_{2}\right)=\mu_{0} \exp \left( \pm 2 \alpha x_{2}\right)$. The family of normalized eigenfunctions is

$$
y_{n}\left(x_{2}\right)=2^{1 / 2} \pi^{-1 / 2} \mu_{0}^{-1 / 2} \exp \left(\mp \alpha x_{2}\right) \sin \left(n x_{2}\right),
$$

and the eigenvalues are $\alpha^{2}+n^{2}$. Hence, in the case where $f\left(x_{2}\right)$ can be written in the form (2.14), we obtain

$$
F\left(x_{1}\right) \geq F(0) \exp \left[-2\left(\alpha^{2}+\sum_{n=1}^{\infty} c_{n}^{2} n^{2} / \sum_{n=1}^{\infty} c_{n}^{2}\right)^{1 / 2} x_{1}\right] .
$$


(b) Case $c=0$. We have that $\mu^{1 / 2}\left(x_{2}\right)=\mu_{0}^{1 / 2}\left(\delta+\alpha x_{2}\right)$, where $\delta=0,1$ and $\alpha$ is an arbitrary positive constant. Thus, $\mu\left(x_{2}\right)=\mu_{0}\left(\delta+\alpha x_{2}\right)^{2}$. The family of normalized eigenfunctions is

$$
y_{n}\left(x_{2}\right)=2^{1 / 2} \pi^{-1 / 2} \mu_{0}^{-1 / 2}\left(\delta+\alpha x_{2}\right)^{-1} \sin \left(n x_{2}\right)
$$

and the eigenvalues are $n^{2}$. If $f\left(x_{2}\right)$ can be written in the form (2.14), we see that

$$
F\left(x_{1}\right) \geq F(0) \exp \left[-2\left(\sum_{n=1}^{\infty} c_{n}^{2} n^{2} / \sum_{n=1}^{\infty} c_{n}^{2}\right)^{1 / 2} x_{1}\right] .
$$

(c) Case $c<0$. In this case we see that $\mu^{1 / 2}\left(x_{2}\right)=\mu_{0}^{1 / 2} \sin \left(\alpha x_{2}+\beta\right)$, where $\alpha^{2}=-c$. If we want that $\mu^{1 / 2}$ is greater than zero, we must impose that $0<\beta<\pi(1-\alpha)$. We have that $\mu\left(x_{2}\right)=\mu_{0} \sin ^{2}\left(\alpha x_{2}+\beta\right)$. The eigenvalues will be $n^{2}-\alpha^{2}$ and the normalized eigenfunctions are

$$
y_{n}=2^{1 / 2} \pi^{-1 / 2} \mu_{0}^{-1 / 2} \sin ^{-1}\left(\alpha x_{2}+\beta\right) \sin \left(n x_{2}\right) .
$$

We note that $\alpha$ is always less than one. Thus, $n^{2}-\alpha^{2}$ is a sequence of positive numbers. Again, when $f\left(x_{2}\right)$ can be expressed as (2.14), we obtain the lower estimate

$$
F\left(x_{1}\right) \geq F(0) \exp \left[-2\left(-\alpha^{2}+\sum_{n=1}^{\infty} c_{n}^{2} n^{2} / \sum_{n=1}^{\infty} c_{n}^{2}\right)^{1 / 2} x_{1}\right] .
$$

When $m^{2} \leq \mu\left(x_{2}\right) \leq M^{2}$, for some positive constants $m$ and $M$ and the function $f\left(x_{2}\right) \in H^{1}[0, \pi]$ can be written as $f\left(x_{2}\right)=\sum_{n} c_{n} \sin \left(n x_{2}\right)$, we have

$$
\frac{\left(\int_{0}^{\pi} \mu\left(x_{2}\right)\left(f^{\prime}\left(x_{2}\right)\right)^{2} d x\right)^{1 / 2}}{\left(\int_{0}^{\pi} \mu\left(x_{2}\right) f^{2}\left(x_{2}\right) d x\right)^{1 / 2}} \leq \frac{\left(\int_{0}^{\pi} M^{2}\left(f^{\prime}\left(x_{2}\right)\right)^{2} d x\right)^{1 / 2}}{\left(\int_{0}^{\pi} m^{2} f^{2}\left(x_{2}\right) d x\right)^{1 / 2}} \leq \frac{M}{m}\left(\frac{\sum_{n=1}^{\infty} c_{n}^{2} n^{2}}{\sum_{n=1}^{\infty} c_{n}^{2}}\right)^{1 / 2} .
$$

So, we have proved the following result:

Corollary 2.3 Let us assume that $m^{2} \leq \mu\left(x_{2}\right) \leq M^{2}$, for some positive constants $m$ and $M$ and that $f\left(x_{2}\right) \in$ $H^{1}[0, \pi]$ can be expressed as $f\left(x_{2}\right)=\sum_{n} c_{n} \sin \left(n x_{2}\right)$. Therefore, the estimate

$$
F\left(x_{1}\right) \geq F(0) \exp \left[-2 \frac{M}{m}\left(\sum_{n=1}^{\infty} c_{n}^{2} n^{2} / \sum_{n=1}^{\infty} c_{n}^{2}\right)^{1 / 2} x_{1}\right]
$$

holds.

\section{Several examples}

The purpose of this section is to illustrate the estimate (2.11) by means of calculations and pictures. We will consider several families of functions $\mu\left(x_{2}\right)$ and we will obtain the values for the right hand side of (2.10). We will denote by $\tau$ the lower bound for the rate of decay given by

$$
\tau=\left(\int_{0}^{L} \mu\left(x_{2}\right)\left(f^{\prime}\left(x_{2}\right)\right)^{2} d x_{2}\right)^{1 / 2} /\left(\int_{0}^{L} \mu\left(x_{2}\right) f^{2}\left(x_{2}\right) d x_{2}\right)^{1 / 2} .
$$


We will see how the estimates depend on the parameters. To make the calculations easier, we assume that $L=\pi$ in this section.

Example 1. We consider the case $f\left(x_{2}\right)=\sin \left(n x_{2}\right), n=1,2,3 \ldots$ and $\mu\left(x_{2}\right)=\mu_{0}\left(1+h\left(x_{2}\right)\right)$, where $\mu_{0}>0$ and $h\left(x_{2}\right)$ is such that

$$
\int_{0}^{\pi} h\left(x_{2}\right) \cos \left(2 n x_{2}\right) d x_{2}=0, n=1,2,3 \ldots
$$

We note that this condition is satisfied whenever $h\left(x_{2}-\pi / 2\right)=-h\left(x_{2}+\pi / 2\right)$ or when $h\left(x_{2}\right)=\alpha x_{2}, \alpha \in \mathbb{R}$. We have that

$$
\int_{0}^{\pi} \mu_{0}\left(1+h\left(x_{2}\right)\right) n^{2} \cos ^{2}\left(n x_{2}\right) d x_{2}=\frac{\mu_{0} n^{2}}{2} \int_{0}^{\pi}\left(1+h\left(x_{2}\right)\right)\left(1+\cos \left(2 n x_{2}\right)\right) d x_{2}=\frac{\mu_{0} n^{2}}{2}\left[\pi+\int_{0}^{\pi} h\left(x_{2}\right) d x_{2}\right] .
$$

On the other side,

$$
\int_{0}^{\pi} \mu_{0}\left(1+h\left(x_{2}\right)\right) \sin ^{2}\left(n x_{2}\right) d x_{2}=\frac{\mu_{0}}{2} \int_{0}^{\pi}\left(1+h\left(x_{2}\right)\right)\left(1-\cos \left(2 n x_{2}\right)\right) d x_{2}=\frac{\mu_{0}}{2}\left[\pi+\int_{0}^{\pi} h\left(x_{2}\right) d x_{2}\right] .
$$

We then see that the right hand side of (2.10) becomes $2 n x_{1}$. So, the decay is inferiorly controlled by $\exp \left(-2 n x_{1}\right)$. Example 2. Now, we take the family $\mu\left(x_{2}\right)=\mu_{0}\left(1+\alpha x_{2}\right)^{k}$, where $\alpha, \mu_{0}>0, k=1,2,3$. If we consider $f\left(x_{2}\right)=\sin \left(n x_{2}\right), n=1,2,3 \cdots$ we could see that

(a) Case $k=1, F^{\prime}(0) / F(0) \geq-2 n$.

(b) Case $k=2, F^{\prime}(0) / F(0) \geq-2 n\left(2 n^{2}(3+\pi \alpha(3+\pi \alpha))+3 \alpha^{2}\right)^{1 / 2}\left(2 n^{2}(3+\pi \alpha(3+\pi \alpha))-3 \alpha^{2}\right)^{-1 / 2}$.

(c) Case $k=3, F^{\prime}(0) / F(0) \geq-2 n\left(n^{2}(2+\pi \alpha(2+\pi \alpha))+3 \alpha^{2}\right)^{1 / 2}\left(n^{2}(2+\pi \alpha(2+\pi \alpha))-3 \alpha^{2}\right)^{-1 / 2}$.

In Figure 1 we represent the lower bound for the rate of decay given by (3.1) for the three above cases when $n=100$. For $k=1, \tau=n$. We can see that for $k=2,3$, if $\alpha$ increases, $\tau$ also increases. Moreover, when $k=3$ the growth is faster than for $k=2$. In fact,

$$
\lim _{\alpha \rightarrow \infty} \tau=n\left(\frac{n^{2} \pi^{2}+3}{n^{2} \pi^{2}-3}\right)^{1 / 2}, k=3 ; \quad \lim _{\alpha \rightarrow \infty} \tau=n\left(\frac{2 n^{2} \pi^{2}+3}{2 n^{2} \pi^{2}-3}\right)^{1 / 2}, k=2 .
$$

When $n=100$, these limits are approximately 100,00304 for $k=3$ and 100,00152 for $k=2$. Figure 2 shows how the lower bound for the rate of decay increases when $n$ increases, for $k=3$ and fixed $\alpha$. In the picture we have represented $\tau$ for three values of $\alpha$. The growth approaches asymptotically to $n$ in a very quick way.

Example 3. Let us consider the functions $\mu\left(x_{2}\right)=\mu_{0} \pi^{-2} x_{2}^{2} \exp \left(2 \alpha x_{2} / \pi\right)$, where $\mu_{0}>0, \alpha \neq 0$ and $f\left(x_{2}\right)=$ $\sin \left(n x_{2}\right), n \in \mathbb{N}$. In this case, we see that

$$
F^{\prime}(0) / F(0) \geq-2 n(A / B)^{1 / 2},
$$

where

$$
A=2 e^{2 \alpha} n^{2} \pi^{2}(5 \alpha-3) \alpha^{5}+n^{4} \pi^{4}\left(e^{2 \alpha}\left(8 \alpha^{2}-4 \alpha+3\right)-3\right) \alpha^{2}+\left(\pi^{6} n^{6}+2 \alpha^{6}\right)\left(e^{2 \alpha}\left(2 \alpha^{2}-2 \alpha+1\right)-1\right)
$$

and

$B=n^{2} \pi^{2}\left[\pi^{4}\left(e^{2 \alpha}(2(\alpha-1) \alpha+1)-1\right) n^{4}+\pi^{2} \alpha^{2}\left(e^{2 \alpha}(4(\alpha-2) \alpha+3)-3\right) n^{2}+2 \alpha^{4}\left(e^{2 \alpha}((\alpha-3) \alpha+3)-3\right)\right]$.

Figures 3 and 4 illustrate the dependence of the lower bound for the rate of decay with respect the parameters, for some values of $\alpha>0$ and $\alpha<0$, respectively. We note that, for fixed $\alpha, \lim _{n \rightarrow \infty}(A / B)=1$ and $\tau$ grows 
asymptotically as $n$. Analogous, Figure 5 shows the dependence of $\tau$ for fixed values of $n$. In this case, for each $n$, the lower bound tends to the asymptote $\tau=\alpha \sqrt{2} / \pi$ as $\alpha \rightarrow \infty$.

Example 4. Set $\mu\left(x_{2}\right)=\mu_{0} \exp \left(2 \alpha x_{2} / \pi\right) \sin ^{2}\left(\beta x_{2} / \pi\right)$, where $\mu_{0}, \beta>0, \alpha \neq 0$ and $f\left(x_{2}\right)=\sin \left(n x_{2}\right), n \in \mathbb{N}$. Therefore,

$$
F^{\prime}(0) / F(0) \geq-2 n\left(A_{1} / B_{1}\right)^{1 / 2}
$$

where

$$
\begin{array}{r}
A_{1}=-\frac{2}{\alpha}-\frac{2 \alpha}{\pi^{2} n^{2}+\alpha^{2}}+\frac{2 \alpha}{\alpha^{2}+\beta^{2}}+\frac{\alpha}{\alpha^{2}+(\beta-n \pi)^{2}}+\frac{\alpha}{\alpha^{2}+(\pi n+\beta)^{2}}+e^{2 \alpha}\left(\frac{2}{\alpha}+\frac{2 \alpha}{\pi^{2} n^{2}+\alpha^{2}}\right) \\
+e^{2 \alpha}\left(\frac{(n \pi-\beta) \sin (2 \beta)-\alpha \cos (2 \beta)}{\alpha^{2}+(\beta-n \pi)^{2}}-\frac{2(\alpha \cos (2 \beta)+\beta \sin (2 \beta))}{\alpha^{2}+\beta^{2}}-\frac{\alpha \cos (2 \beta)+(\pi n+\beta) \sin (2 \beta)}{\alpha^{2}+(\pi n+\beta)^{2}}\right)
\end{array}
$$

and

$$
\begin{gathered}
B_{1}=-\frac{2}{\alpha}+\frac{2 \alpha}{\pi^{2} n^{2}+\alpha^{2}}+\frac{2 \alpha}{\alpha^{2}+\beta^{2}}-\frac{\alpha}{\alpha^{2}+(\beta-n \pi)^{2}}-\frac{\alpha}{\alpha^{2}+(\pi n+\beta)^{2}}+e^{2 \alpha}\left(\frac{2}{\alpha}-\frac{2 \alpha}{\pi^{2} n^{2}+\alpha^{2}}\right) \\
+e^{2 \alpha}\left(\frac{\alpha \cos (2 \beta)-(n \pi-\beta) \sin (2 \beta)}{\alpha^{2}+(\beta-n \pi)^{2}}-\frac{2(\alpha \cos (2 \beta)+\beta \sin (2 \beta))}{\alpha^{2}+\beta^{2}}+\frac{\alpha \cos (2 \beta)+(\pi n+\beta) \sin (2 \beta)}{\alpha^{2}+(\pi n+\beta)^{2}}\right) .
\end{gathered}
$$

In Figure 6 we have represented $\tau$ for different values of $n$, with $\beta=1 / 2$. The lower bound tends to the asymptote $\tau=\alpha \sqrt{2} / \pi$ as $\alpha \rightarrow \infty$. Moreover, for $\beta=1 / 2, \tau$ grows asymptotically as $n$ when $n \rightarrow \infty$ for fixed $\alpha$ (see Figures 7 and 8 , for $\alpha>0$ and $\alpha<0$, respectively).

Example 5. Set $\mu\left(x_{2}\right)=\mu_{0} \alpha^{-2}\left[1-\exp \left(-\alpha x_{2} / \pi\right)\right]^{2}$, where $\mu_{0}, \alpha>0$ and $f\left(x_{2}\right)=\sin \left(n x_{2}\right), n \in \mathbb{N}$. Then,

$$
\frac{F^{\prime}(0)}{F(0)} \geq-2 n\left(\frac{4 \pi^{4}\left(M+4 e^{\alpha}-1\right) n^{4}+\pi^{2} \alpha^{2}\left(5 M+24 e^{\alpha}-9\right) n^{2}+2\left(e^{2 \alpha}(\alpha-3)+4 e^{\alpha}-1\right) \alpha^{4}}{2 e^{2 \alpha} \alpha^{5}+n^{2} \pi^{2}\left(5 M+16 e^{\alpha}-1\right) \alpha^{2}+4 n^{4} \pi^{4}\left(M+4 e^{\alpha}-1\right)}\right)^{1 / 2}
$$

with $M=e^{2 \alpha}(2 \alpha-3)$. Here, the lower bound $\tau$ increases as $n$ when $n \rightarrow \infty$. On the other hand, $\tau \sim n$ as $\alpha \rightarrow \infty$. Figures 9 and 10 illustrates this condition when $n=90$ and $n=800$, respectively.

Example 6. Set $\mu\left(x_{2}\right)=\mu_{0} \sinh ^{2}\left(\beta x_{2} / L\right)$ for $\mu_{0}, \beta>0$ and $f\left(x_{2}\right)=\sin \left(n x_{2}\right)$. Therefore,

$$
\frac{F^{\prime}(0)}{F(0)} \geq-2 n\left(\frac{\beta^{3}+n^{2} \pi^{2} \beta-\left(\pi^{2} n^{2}+2 \beta^{2}\right) \cosh (\beta) \sinh (\beta)}{\beta^{3}+n^{2} \pi^{2} \beta-n^{2} \pi^{2} \cosh (\beta) \sinh (\beta)}\right)^{1 / 2}
$$

For fixed $\beta$, the lower bound $\tau \sim n$ as $n \rightarrow \infty$ (see Figure 11). On the other hand, for each $n$, the lower bound of the rate of decay tends to the asymptote $\tau=\beta \sqrt{2} / \pi$ as $\beta \rightarrow \infty$ (see Figure 12).

\section{A change of variable}

In this section we consider the general case where $\mu$ is a $C^{1}$-function which can depend on the both variables $x_{1}$ and $x_{2}$ and such that $\mu\left(x_{1}, x_{2}\right)>0$. We here are interested in the solutions of the problem (1.1)-(1.3), when $f\left(x_{2}\right) \in H^{1,2}[0, L]$. However, because of technical reasons, we also assume some extra asymptotic conditions ${ }^{2}$

$$
\mu^{1 / 2} u \rightarrow 0, \mu^{1 / 2} u_{, i} \rightarrow 0 \text { and } \mu^{-1 / 2} \mu_{, i} u \rightarrow 0 \text { as } x_{1} \rightarrow \infty \text { (uniformly on } x_{2} \text { ) }
$$

and

$$
\left.\mu^{1 / 2} \Delta\left(\mu^{1 / 2}\right) u^{2} \rightarrow 0 \text { as } x_{1} \rightarrow \infty \text { (uniformly on } x_{2}\right) .
$$

\footnotetext{
${ }^{2}$ The proposed conditions should be seen in the sense that the shear modulus is a data and the class of solutions we will work are restricted to satisfy the asymptotic conditions. In the examples that we will consider, condition (1.3) imply (4.1) and (4.2).
} 
The analysis in this situation starts by considering the change of variables

$$
u\left(x_{1}, x_{2}\right)=U\left(x_{1}, x_{2}\right) \mu^{-1 / 2}\left(x_{1}, x_{2}\right) .
$$

So, the function $U\left(x_{1}, x_{2}\right)$ satisfies the equation

$$
\Delta U+\Gamma(\mu) U=0, \text { where } \Gamma(\mu)=\frac{1}{4 \mu^{2}}\left[|\nabla \mu|^{2}-2 \mu \Delta \mu\right]=-\mu^{-1 / 2} \Delta\left(\mu^{1 / 2}\right) .
$$

We note that the conditions (4.1) imply that

$$
U \rightarrow 0 \text { and } U_{, i} \rightarrow 0 \text { as } x_{1} \rightarrow \infty .
$$

We also note that

$$
U\left(x_{1}, 0\right)=0, U\left(x_{1}, L\right)=0, x_{1} \geq 0
$$

and

$$
U\left(0, x_{2}\right)=\mu^{1 / 2}\left(0, x_{2}\right) f\left(x_{2}\right), x_{2} \in[0, L] .
$$

Multiplying the equation (4.4) by $U_{, 1}$ and integrating over $\left[x_{1}, x_{1}+h\right] \times[0, L]$ we get that

$$
\int_{0}^{L} \int_{x_{1}}^{x_{1}+h} \frac{d}{d \xi_{1}}\left(\frac{1}{2} U_{, 1}^{2}-\frac{1}{2} U_{, 2}^{2}+\frac{1}{2} \Gamma U^{2}\right) d \xi_{1} d x_{2}-\int_{0}^{L} \int_{x_{1}}^{x_{1}+h} \frac{1}{2} \Gamma_{, 1} U^{2} d \xi_{1} d x_{2}=0 .
$$

Hence, we obtain the energy equation

$$
E\left(x_{1}+h\right)-E\left(x_{1}\right)=D\left(x_{1}, x_{1}+h\right)
$$

where

$$
E\left(x_{1}\right)=\frac{1}{2} \int_{0}^{L}\left(U_{, 1}^{2}+\Gamma U^{2}-U_{, 2}^{2}\right) d x_{2} \text { and } D\left(x_{1}, x_{1}+h\right)=\frac{1}{2} \int_{0}^{L} \int_{x_{1}}^{x_{1}+h} \Gamma_{, 1} U^{2} d \xi_{1} d x_{2} .
$$

Our next step is to use again the logarithmic convexity argument. However, we will need to restrict our attention to a subclass of functions $\mu\left(x_{1}, x_{2}\right)$. We will assume that $\Gamma_{, 1} \geq 0$ for every $\left(x_{1}, x_{2}\right)$. Therefore, $D \geq 0$. It then follows that $E\left(x_{1}\right) \leq E\left(x_{1}+h\right)$, for every $h \geq 0$. In view of the asymptotic condition (4.2), that is, $\Gamma U^{2} \rightarrow 0$ as $x_{1} \rightarrow 0$, and condition (1.3) it follows that $\lim _{h \rightarrow \infty} E\left(x_{1}+h\right)=0$, for every $x_{1} \geq 0$. So, we obtain that $E\left(x_{1}\right) \leq 0$, for every $x_{1} \geq 0$. From the energy equation we get

$$
\int_{0}^{L} U_{, 2}^{2} d x_{2} \geq \int_{0}^{L}\left(U_{, 1}^{2}+\Gamma U^{2}\right) d x_{2}
$$

With the help of this inequality, we can reproduce the logarithmic convexity argument. We define

$$
F\left(x_{1}\right)=\frac{1}{2} \int_{0}^{L} U^{2} d x_{2}
$$

Hence,

$$
F^{\prime}\left(x_{1}\right)=\int_{0}^{L} U U_{, 1} d x_{2} \text { and } F^{\prime \prime}\left(x_{1}\right)=\int_{0}^{L} U_{, 1} U_{, 1} d x_{2}+\int_{0}^{L} U U_{, 11} d x_{2} .
$$

From the equation (4.4) we obtain

$$
F^{\prime \prime}\left(x_{1}\right)=\int_{0}^{L} U_{, 1}^{2} d x_{2}+\int_{0}^{L} U_{, 2}^{2} d x_{2}-\int_{0}^{L} \Gamma U^{2} d x_{2} \geq 2 \int_{0}^{L} U_{, 1}^{2} d x_{2} .
$$


Therefore,

$$
F F^{\prime \prime}-\left(F^{\prime}\right)^{2} \geq\left(\int_{0}^{L} U^{2} d x_{2}\right)\left(\int_{0}^{L} U_{, 1}^{2} d x_{2}\right)-\left(\int_{0}^{L} U U_{, 1} d x_{2}\right)^{2} .
$$

If we recall Hölder inequality, we find that $F F^{\prime \prime}-\left(F^{\prime}\right)^{2} \geq 0$, i.e., $\ln F\left(x_{1}\right)$ is a convex function and so (see [2], p.19)

$$
F\left(x_{1}\right) \geq F(0) \exp \left(\left(F^{\prime}(0) / F(0)\right) x_{1}\right) .
$$

The decay rates for $F\left(x_{1}\right)$ is controlled by $F^{\prime}(0) / F(0)$. Thus, we need to obtain an estimate for this quotient. We have that

$$
-F^{\prime}(0) \leq\left(\int_{0}^{L} U^{2}\left(0, x_{2}\right) d x_{2}\right)^{1 / 2}\left(\int_{0}^{L} U_{, 1}^{2}\left(0, x_{2}\right) d x_{2}\right)^{1 / 2}
$$

which implies the bound

$$
-F^{\prime}(0) \leq\left(\int_{0}^{L} U^{2}\left(0, x_{2}\right) d x_{2}\right)^{1 / 2}\left(\int_{0}^{L} U_{, 2}^{2}\left(0, x_{2}\right) d x_{2}-\int_{0}^{L} \Gamma\left(0, x_{2}\right) U^{2}\left(0, x_{2}\right) d x_{2}\right)^{1 / 2} .
$$

Thus, we obtain the estimate

$$
\frac{F^{\prime}(0)}{F(0)} \geq-2\left(\int_{0}^{L} U_{, 2}^{2}\left(0, x_{2}\right) d x_{2}-\int_{0}^{L} \Gamma\left(0, x_{2}\right) U^{2}\left(0, x_{2}\right) d x_{2}\right)^{1 / 2}\left(\int_{0}^{L} U^{2}\left(0, x_{2}\right) d x_{2}\right)^{-1 / 2} .
$$

The change of variable (4.3) yields

$$
F^{\prime}(0) / F(0) \geq-2 A^{*} / B^{*}
$$

where

$$
A^{*}=\left[\int_{0}^{L}\left(\frac{1}{2} \mu^{-1 / 2}\left(0, x_{2}\right) \mu_{, 2}\left(0, x_{2}\right) f\left(x_{2}\right)+\mu^{1 / 2}\left(0, x_{2}\right) f^{\prime}\left(x_{2}\right)\right)^{2} d x_{2}-\int_{0}^{L} \Gamma\left(0, x_{2}\right) f^{2}\left(x_{2}\right) \mu\left(0, x_{2}\right) d x_{2}\right]^{1 / 2}
$$

and

$$
B^{*}=\left(\int_{0}^{L} f^{2}\left(x_{2}\right) \mu\left(0, x_{2}\right) d x_{2}\right)^{1 / 2} .
$$

However, the function $F\left(x_{1}\right)$ has been only a tool for our study. In fact, our aim is to obtain estimates for the function $u\left(x_{1}, x_{2}\right)$. For this reason we define the function

$$
G\left(x_{1}\right)=\frac{1}{2} \int_{0}^{L} u^{2} d x_{2}=\frac{1}{2} \int_{0}^{L} U^{2} \mu^{-1} d x_{2} .
$$

Set $\delta^{+}\left(x_{1}\right)=\inf _{x_{2} \in[0, L]} \mu^{-1}\left(x_{1}, x_{2}\right)$. Therefore, we get

$$
G\left(x_{1}\right) \geq \delta^{+}\left(x_{1}\right) F\left(x_{1}\right) \geq \delta^{+}\left(x_{1}\right) F(0) \exp \left[\left(F^{\prime}(0) / F(0)\right) x_{1}\right],
$$

which allows us to obtain the estimate

$$
G\left(x_{1}\right) \geq \delta^{+}\left(x_{1}\right) F(0) \exp \left[-2\left(A^{*} / B^{*}\right) x_{1}\right] .
$$

Thus, we have prove the following result.

Theorem 4.1 Let $u\left(x_{1}, x_{2}\right)$ be a solution of the problem determined by (1.1)-(1.2) subject to the asymptotic conditions (1.3) and (4.1)-(4.2). Let us also assume that $\Gamma_{, 1} \geq 0$, where $\Gamma$ is given by (4.4). Then, the estimate (4.25) is satisfied, where $A^{*}$ and $B^{*}$ are given by (4.21) and (4.22), respectively. 
Remark 4.1 In the case when $\mu$ does not depend on $x_{2}$, we see that $\delta^{*}\left(x_{1}\right)=\mu^{-1}\left(x_{1}\right)$ and $F(0)=\frac{\mu(0)}{2} \int_{0}^{L} f^{2}\left(x_{2}\right) d x_{2}$. So, estimate (4.25) becomes

$$
G\left(x_{1}\right) \geq \mu^{-1}\left(x_{1}\right) \frac{\mu(0)}{2}\left(\int_{0}^{L} f^{2}\left(x_{2}\right) d x_{2}\right) \exp \left[-2\left(\frac{\int_{0}^{L}\left[\left(f^{\prime}\left(x_{2}\right)\right)^{2}-\Gamma(0) f^{2}\left(x_{2}\right)\right] d x_{2}}{\int_{0}^{L} f^{2}\left(x_{2}\right) d x_{2}}\right)^{1 / 2} x_{1}\right] .
$$

It is also possible to obtain an impossibility of localization type result.

Corollary 4.1 Let us to assume that $\mu\left(x_{1}, x_{2}\right) \geq 0, \Gamma_{, 1}\left(x_{1}, x_{2}\right) \geq 0 ; \mu\left(0, x_{2}\right)$ and $\Gamma\left(0, x_{2}\right)$ are upper bounded by a constant; $f\left(x_{2}\right)$ and $f^{\prime}\left(x_{2}\right)$ are bounded. Then, the only solution which vanishes identically after a finite time is the null solution. That is the case when $f\left(x_{2}\right) \equiv 0$.

Our next step is to obtain several precise estimates when we select suitable functions $\mu\left(x_{1}, x_{2}\right)$ and for initial data of the type $f\left(x_{2}\right)=\sin \left(n x_{2}\right), n=1,2,3 \ldots$ We will take $L=\pi$ in all our examples.

We first restrict our attention to the case $\mu=\mu\left(x_{1}\right)$.

Example 7: Let us consider $\mu\left(x_{1}\right)=\mu_{0}\left(1+\alpha x_{1}\right)^{-k}$, where $k>0, \alpha>0, \mu_{0}>0$. Clearly, $\mu^{1 / 2}$ and $\mu^{-1 / 2} \mu_{, i}$ are bounded. Moreover, $\mu^{1 / 2} \Delta\left(\mu^{1 / 2}\right)=\mu_{0} \alpha^{2} k(k+2) 2^{-1}\left(1+\alpha x_{1}\right)^{-(k+2)}$ is also bounded. So, whenever (1.3) holds, conditions (4.1) and (4.2) are also satisfied. We note that

$$
\Gamma=-k(k+2) \alpha^{2} 4^{-1}\left(1+\alpha x_{1}\right)^{-2} \text { and } \Gamma_{, 1}=\alpha^{3} k(k+2) 2^{-1}\left(1+\alpha x_{1}\right)^{-3}>0 .
$$

We apply the estimate (4.26) when $f\left(x_{2}\right)=\sin \left(n x_{2}\right), n=1,2,3 \ldots$ and we obtain that

$$
G\left(x_{1}\right) \geq \frac{\pi}{4}\left(1+\alpha x_{1}\right)^{k} \exp \left(-\sqrt{4 n^{2}+k(k+2) \alpha^{2}} x_{1}\right) .
$$

So, the decay is asymptotically controlled by $\exp \left(-\sqrt{4 n^{2}+k(k+2) \alpha^{2}} x_{1}\right)$.

Example 8: Let us consider $\mu\left(x_{1}\right)=\mu_{0} \exp \left(-\alpha x_{1}\right)$, for $\mu, \alpha>0$. The functions $\mu^{1 / 2}, \mu^{-1 / 2} \mu_{i, i}$ and $\mu^{1 / 2} \Delta\left(\mu^{1 / 2}\right)=$ $\mu_{0} \alpha^{2} 4^{-1} \exp \left(-\alpha x_{1}\right)$ are bounded. Then, from (1.3) we get (4.1) and (4.2). Furthermore $\Gamma=\frac{-\alpha^{2}}{4}$ and so $\Gamma_{, 1}=0$, $\forall x_{1}$. When $f\left(x_{2}\right)=\sin \left(n x_{2}\right)$, it follows

$$
G\left(x_{1}\right) \geq \frac{\pi}{4} \exp \left(\alpha x_{1}\right) \exp \left(-\sqrt{4 n^{2}+\alpha^{2}} x_{1}\right)=\frac{\pi}{4} \exp \left[\left(\alpha-\sqrt{4 n^{2}+\alpha^{2}}\right) x_{1}\right] .
$$

Here, the decay is controlled by $\exp \left[\left(\alpha-\sqrt{4 n^{2}+\alpha^{2}}\right) x_{1}\right]$.

Now we consider several examples for the general case $\mu=\mu\left(x_{1}, x_{2}\right)$.

Example 9: Let us consider $\mu\left(x_{1}, x_{2}\right)=\mu_{0}\left(1+\alpha x_{1}\right)^{-k} \exp \left(\beta x_{2}\right)$, where $\mu_{0}, k, \alpha>0$ and $\beta \neq 0$ and assume $f\left(x_{2}\right)=\sin \left(n x_{2}\right)$. Clearly, $\mu^{1 / 2}$ and $\mu^{-1 / 2} \mu_{, i}$ are bounded. Moreover,

$$
\mu^{1 / 2} \Delta\left(\mu^{1 / 2}\right)=\mu_{0} \exp \left(\beta x_{2}\right)\left[\alpha k(k+2) 2^{-1}\left(1+\alpha x_{1}\right)^{-(k+2)}+\beta^{2} 4^{-1}\left(1+\alpha x_{1}\right)^{-k}\right]
$$

is also bounded. Thus, if (1.3) holds, then (4.1) and (4.2) also hold. We get

$$
\Gamma=-4^{-1}\left[\beta^{2}+\alpha^{2} k(k+2)\left(1+\alpha x_{1}\right)^{-2}\right] \text { and } \Gamma_{, 1}=2^{-1} \alpha^{3} k(k+2)\left(1+\alpha x_{1}\right)^{-3}>0 .
$$

In this case,

$$
\delta^{+}\left(x_{1}\right)= \begin{cases}\mu_{0}^{-1}\left(1+\alpha x_{1}\right)^{k} \exp (-\beta \pi), & \text { if } \beta>0 \\ \mu_{0}^{-1}\left(1+\alpha x_{1}\right)^{k}, & \text { if } \beta<0\end{cases}
$$


Moreover, $F(0)=\mu_{0}\left(e^{\pi \beta}-1\right) n^{2}\left(4 n^{2} \beta+\beta^{3}\right)^{-1}$. Direct calculations give us

$$
A=\mu_{0}^{1 / 2}\left(\frac{\left(e^{\pi \beta}-1\right) n^{2}\left(4 n^{2}+k(k+2) \alpha^{2}+2 \beta^{2}\right)}{2\left(4 n^{2} \beta+\beta^{3}\right)}\right)^{1 / 2} \text { and } B=\mu_{0}^{1 / 2}\left(\frac{2\left(e^{\pi \beta}-1\right) n^{2}}{4 n^{2} \beta+\beta^{3}}\right)^{1 / 2} \text {. }
$$

Therefore, estimate (4.25) becomes

$$
G\left(x_{1}\right) \geq \frac{\left(1-e^{-\pi \beta}\right) n^{2}}{4 n^{2} \beta+\beta^{3}}\left(1+\alpha x_{1}\right)^{k} \exp \left[-\sqrt{4 n^{2}+k(k+2) \alpha^{2}+2 \beta^{2}} x_{1}\right], \text { if } \beta>0
$$

and

$$
G\left(x_{1}\right) \geq \frac{\left(e^{\pi \beta}-1\right) n^{2}}{4 n^{2} \beta+\beta^{3}}\left(1+\alpha x_{1}\right)^{k} \exp \left[-\sqrt{4 n^{2}+k(k+2) \alpha^{2}+2 \beta^{2}} x_{1}\right], \text { if } \beta<0 .
$$

In both cases, $\beta \neq 0$, the decay is controlled by $\exp \left[-\sqrt{4 n^{2}+k(k+2) \alpha^{2}+2 \beta^{2}} x_{1}\right]$.

Example 10: Let us consider $\mu\left(x_{1}, x_{2}\right)=\mu_{0} \exp \left(-\alpha x_{1}+\beta x_{2}\right)$, where $\mu_{0}, \alpha>0$ and $\beta \neq 0$. The functions $\mu^{1 / 2}$ and $\mu^{-1 / 2} \mu_{, i}$ are bounded. Here, $\mu^{1 / 2} \Delta\left(\mu^{1 / 2}\right)=\mu_{0} 4^{-1}\left(\alpha^{2}+\beta^{2}\right) \exp \left(-\alpha x_{1}+\beta x_{2}\right)$ is bounded and so, all our requirements are accomplished. We study estimate (4.25) when $f\left(x_{2}\right)=\sin \left(n x_{2}\right)$. We have $\Gamma=\frac{-\left(\alpha^{2}+\beta^{2}\right)}{4}$ and $\Gamma_{, 1}=0$. Now,

$$
G\left(x_{1}\right) \geq \frac{\left(1-e^{-\pi \beta}\right) n^{2}}{4 n^{2} \beta+\beta^{3}} \exp \left[\left(\alpha-\sqrt{4 n^{2}+\alpha^{2}+2 \beta^{2}}\right) x_{1}\right] .
$$

Hence, the decay is controlled by $\exp \left[\left(\alpha-\sqrt{4 n^{2}+\alpha^{2}+2 \beta^{2}}\right) x_{1}\right]$.

\section{References}

[1] Aboudi, J., Pindera, M.J., Arnold, S.M., Higher order theory for functionally graded materials. Composites: Part B, 30 (1999) 777-832.

[2] Ames, K.A., Straughan, B., Non-standard and improperly posed problems. Academic Press, San Diego (1997)

[3] Borrelli, A., Horgan, C.O., Patria, M.C., Exponential decay of end effects in anti-plane shear for functionally graded piezoelectric materials. Proceedings Royal Society London A, 460 (2004) 1193-1212.

[4] Chan, A.M., Horgan, C.O., End effects in anti-plane shear for an inhomogeneous isotropic linearly elastic semi-infinite strip. Journal of Elasticity, 51 (1998) 227-242.

[5] Erdogan, F., Fracture mechanics for functionally graded materials. Composites Engineering, 5 (1995) 753-770.

[6] Horgan, C.O., Payne, L.E., On the asymptotic behavior of solutions of linear second-order boundary value problems on a semi-infinite strip. Archive of Rational Mechanics and Analysis, 124 (1993) 277-303.

[7] Horgan, C.O., Quintanilla, R., Saint-Venant end effects in antiplane shear for functionally graded linearly elastic materials. Mathematics and Mechanics of Solids, 6 (2001a) 115-132.

[8] Horgan, C.O., Quintanilla, R., Spatial decay of transient end effects in functionally graded heat conducting materials. Quarterly of Applied Mathematics, 59 (2001b) 529-542.

[9] Leseduarte, M.C., Quintanilla, R., Saint-Venant decay rates for a non-homogeneous isotropic mixture of elastic solids in anti-plane shear. International Journal of Solids and Structures, 42 (2005) 2977-3000.

[10] Leseduarte, M.C., Quintanilla, R., Saint-Venant decay rates for an anisotropic and non-homogeneous mixture of elastic solids in anti-plane shear. International Journal of Solids and Structures, 45 (2008) 1697-1712. 
[11] Pindera, M.J., Aboudi, J., Glasser, A.M., Arnold, S.M., (eds.), Use of composite in multi-phased and functionally graded materials. Special issue of Composites, Part B: Engineering, 28B (1997).

[12] Scalpato, M.R., Horgan, C.O., Saint-Venant decay rate for an isotropic inhomogeneous linearly elastic solid in anti-plane shear. Journal of Elasticity, 48 (1997) 145-166.

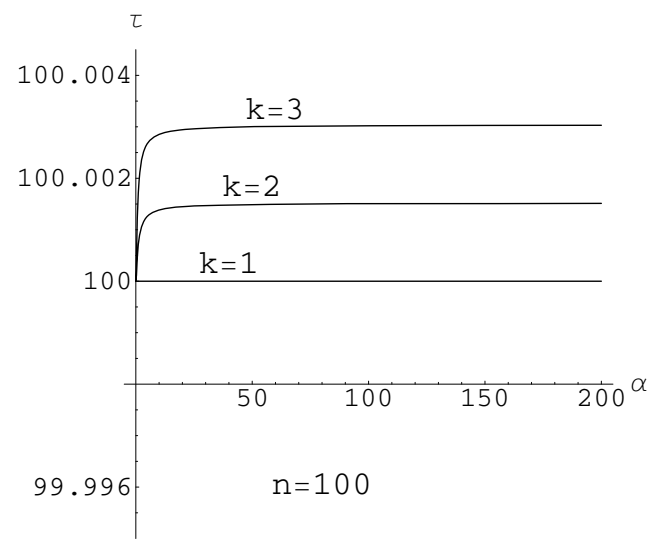

Figure 1: Lower bounds for $\mu\left(x_{2}\right)=\mu_{0}\left(1+\alpha x_{2}\right)^{k}$ when $n=100$.

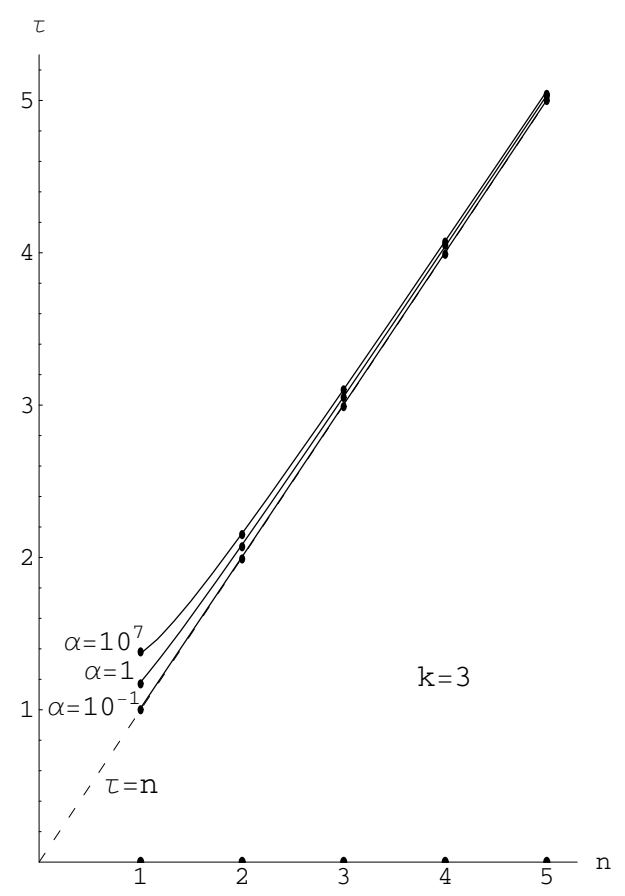

Figure 2: Lower bounds for $\mu\left(x_{2}\right)=\mu_{0}\left(1+\alpha x_{2}\right)^{k}$ with $k=3$. 


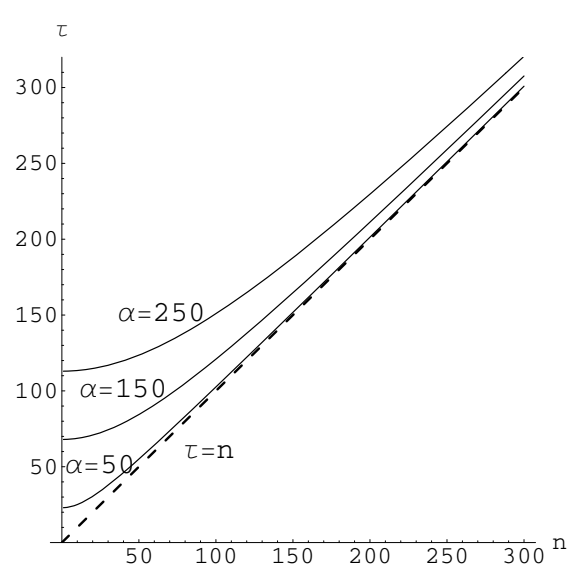

Figure 3: Lower bounds for $\mu\left(x_{2}\right)=\mu_{0} \pi^{-2} x_{2}^{2} \exp \left(2 \alpha x_{2} / \pi\right)$ when $\alpha>0$.

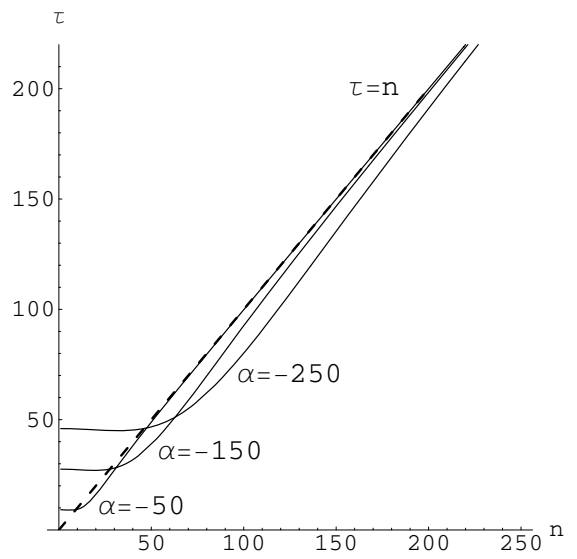

Figure 4: Lower bounds for $\mu\left(x_{2}\right)=\mu_{0} \pi^{-2} x_{2}^{2} \exp \left(2 \alpha x_{2} / \pi\right)$ when $\alpha<0$.

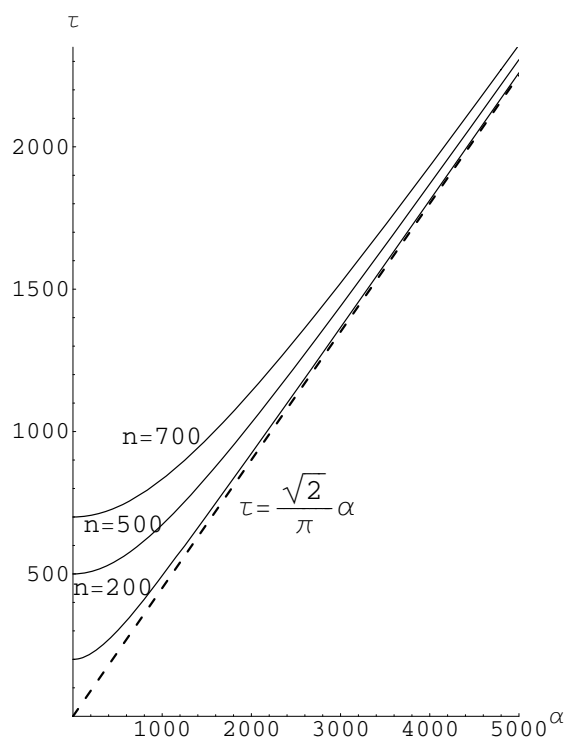

Figure 5: Lower bounds for $\mu\left(x_{2}\right)=\mu_{0} \pi^{-2} x_{2}^{2} \exp \left(2 \alpha x_{2} / \pi\right)$. 


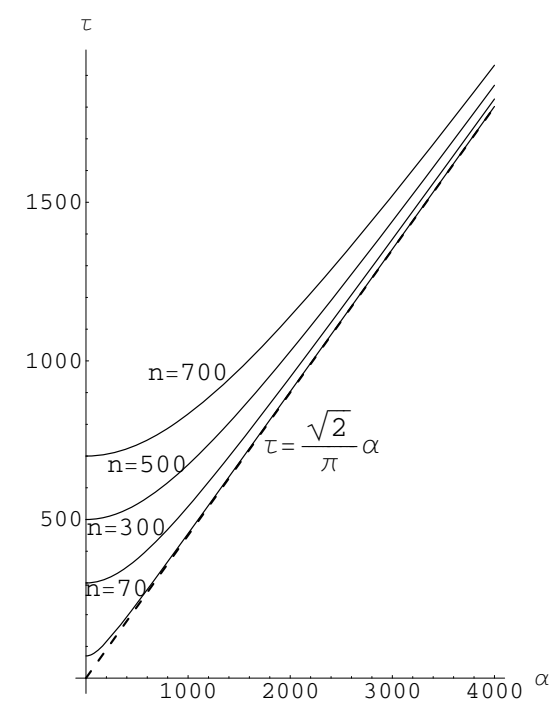

Figure 6: Lower bounds for $\mu\left(x_{2}\right)=\mu_{0} \exp \left(2 \alpha x_{2} / \pi\right) \sin ^{2}\left(\beta x_{2} / \pi\right)$ with $\beta=1 / 2$.

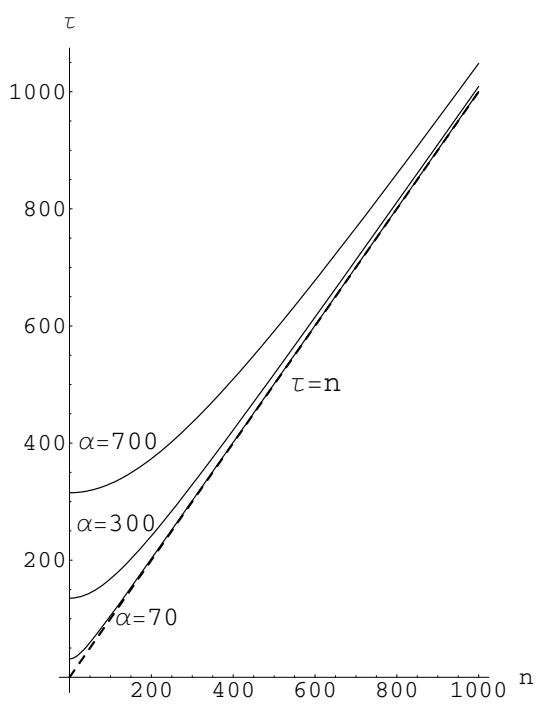

Figure 7: Lower bounds for $\mu\left(x_{2}\right)=\mu_{0} \exp \left(2 \alpha x_{2} / \pi\right) \sin ^{2}\left(\beta x_{2} / \pi\right)$ with $\beta=1 / 2$ when $\alpha>0$. 


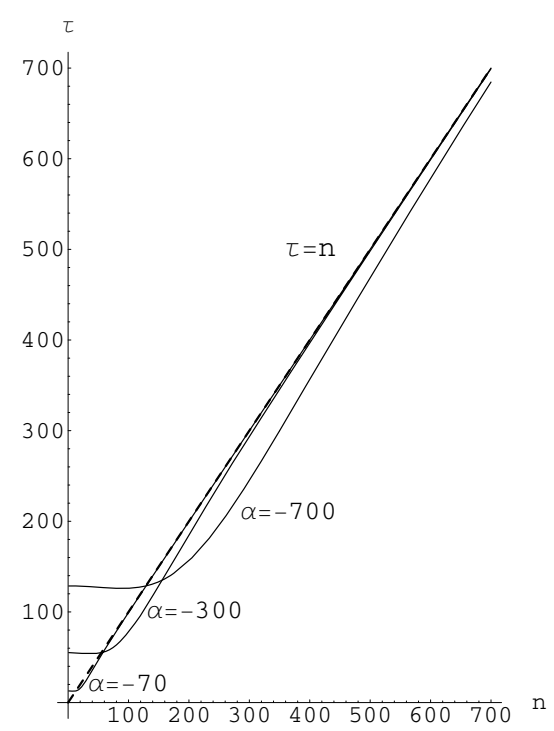

Figure 8: Lower bounds for $\mu\left(x_{2}\right)=\mu_{0} \exp \left(2 \alpha x_{2} / \pi\right) \sin ^{2}\left(\beta x_{2} / \pi\right)$ with $\beta=1 / 2$ when $\alpha<0$.

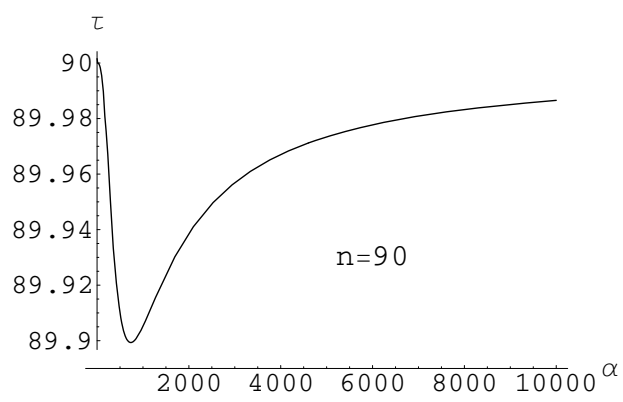

Figure 9: Lower bounds for $\mu\left(x_{2}\right)=\mu_{0} \alpha^{-2}\left[1-\exp \left(-\alpha x_{2} / \pi\right)\right]^{2}$.

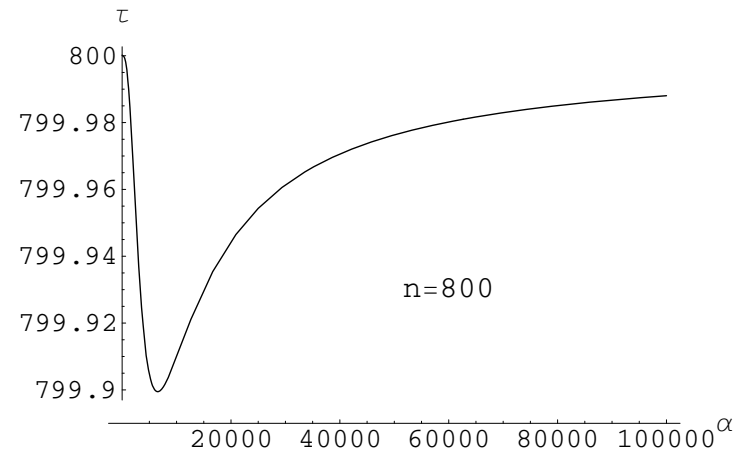

Figure 10: Lower bounds for $\mu\left(x_{2}\right)=\mu_{0} \alpha^{-2}\left[1-\exp \left(-\alpha x_{2} / \pi\right)\right]^{2}$. 


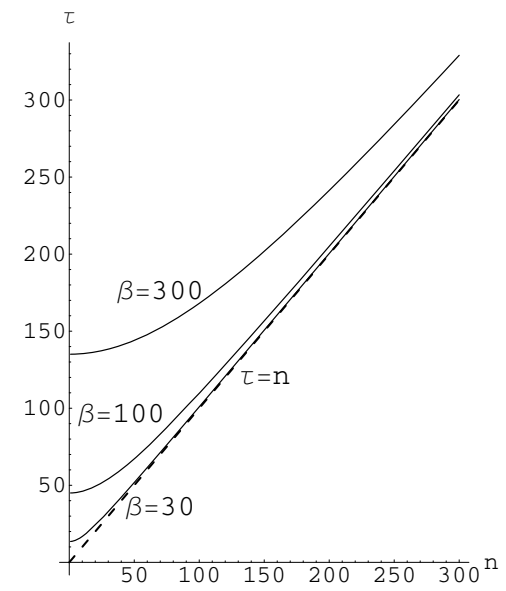

Figure 11: Lower bounds for $\mu\left(x_{2}\right)=\mu_{0} \sinh ^{2}\left(\beta x_{2} / \pi\right)$.

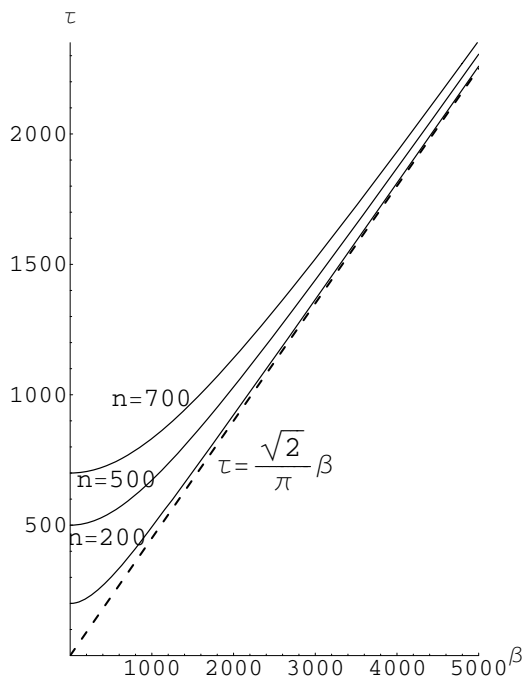

Figure 12: Lower bounds for $\mu\left(x_{2}\right)=\mu_{0} \sinh ^{2}\left(\beta x_{2} / \pi\right)$. 